the city. The routine information on participation was used to compare the characteristics of participants for the five events. The qualitative findings were analysed thematically to identify similarities and differences between the different events which might explain significant differences in numbers and characteristics of participants across the five different events.

Results Index of multiple deprivation was the main predictor of attendance numbers, with weekly attendance varying from 584 (most affluent location) to 63 (most deprived location). Men outnumbered women (57\% vs $43 \%$ ) and ethnic diversity was low across all events. Significant differences in participant experience were observed between the larger and smaller events in terms of the competitive ethos, degree of anonymity, sense of community and social engagement. There appeared to be a trade-off with larger events offering anonymity and a spirt of competition, whilst smaller events seemed to encourage a more supportive and friendly social experience for participants.

Conclusion Patterns of participation suggest that mass weekly community events of this nature are, whilst likely to provide health benefits to the population as a whole, also likely to be responsible for significant intervention generated inequalities in uptake of opportunities for physically active recreation. Current efforts to increase access to opportunities for physical activity in deprived communities may need to consider carefully how to identify effective ways to attract more participants whilst preserving the perceived benefits of smaller events that promote community ownership and engagement.

\section{P3 AREA DEPRIVATION MEASURES IN BRAZIL: A SCOPING REVIEW}

${ }^{1,2} \mathrm{~A}$ Ferreira* ${ }^{1,3} \mathrm{MY}$ Ichihara, 'D Ramos, ${ }^{1,2} \mathrm{P}$ Rebouças, ${ }^{1,2} \mathrm{FJ}$ Oliveira, ${ }^{1,2} \mathrm{C}$ Teixeira, ${ }^{3,4} \mathrm{SV}$ Katikireddi, ${ }^{1,2,3} \mathrm{M}$ Barreto, ${ }^{3,4} \mathrm{AH}$ Leyland, ${ }^{3,4} \mathrm{R}$ Dundas. ${ }^{1}$ Center for Data Integration and Health Knowledge, Fiocruz Bahia, Salvador, Brazili, ${ }^{2}$ Graduate School of Public Health, Federal University of Bahia, Salvador, Brazil; ${ }^{3}$ NIHR Global Health Research Group on Social Policy and Health Inequalities, University of Glasgow, Glasgow, UK; ${ }^{4}$ Social and Public Health Sciences Unit, MRC/CSO, Glasgow, UK

\subsection{6/jech-2018-SSMabstracts. 129}

Background Brazil is a country with high rates of inequality. Although overall poverty rates have declined and global measures of socioeconomic conditions have shown consistent improvements in recent decades, internal disparities remain substantial. Area-based deprivation indices are important for understanding social inequalities. The purpose of this review is to inform the development of a small area deprivation index for Brazil, describing and assessing currently used area based measures of socioeconomic inequalities in Brazil for health research.

Methods We searched five electronic databases and seven websites of Brazilian research institutions and governmental agencies. Inclusion criteria were multiple measures of deprivation, small areas (i.e. finer geography than country-level) in Brazil. Studies had to be published in English, Portuguese or Spanish. We extracted data on study characteristics, name of the deprivation measure, area level, geographical coverage, variables used to calculate the index and whether it was used to report a health outcome. Results were tabulated according to the area-level used and dimensions of deprivation or poverty included in the measures. We used a narrative synthesis approach to summarise the different deprivation measures available, highlighting their strengths and weaknesses for application to public health research.

Results A total of 7199 records were retrieved, 126 full text articles were assessed using the inclusion criteria and a final list of 30 articles were selected. Most of the studies were excluded as they did not focus on area level measurements $(n=69)$. We identified no small area deprivation measures that have been applied to the whole of Brazil. Three studies did cover the entire country of Brazil but the 'small areas' used were municipalities with an average population of 37000 . We found limited deprivation measures using the census tract area level and few measures using the most recent 2010 Census. Papers were grouped into six dimensions: income, education, sanitation, household conditions, ethnicity and others. These measures were mainly used to study infectious and parasitic diseases. Few studies used the measures to assess inequalities in mortality and no studies used the deprivation measure to evaluate the impact of social programs.

Conclusion Currently there is no up-to-date small area-based deprivation measure that covers the whole of Brazil. Areabased deprivation indicators have been in use in the UK and other countries for over 30 years. There is a need to develop a similar small-area deprivation index for Brazil that can be used to measure and monitor inequalities in health and mortality.

\section{P4 COMMUNITY FOOD PRODUCTION IN SMALL ISLAND DEVELOPING STATES: A SYSTEMATIC SCOPING REVIEW OF HEALTH, SOCIAL, ECONOMIC AND ENVIRONMENTAL IMPACTS}

${ }^{1}$ E Haynes, ${ }^{2} \mathrm{C}$ Brown ${ }^{*},{ }^{3} \mathrm{C}$ Wou, ${ }^{4} \mathrm{C}$ Vogliano, ${ }^{1} \mathrm{C}$ Guell ${ }^{*},{ }^{3} \mathrm{~N}$ Unwin. ${ }^{1}$ European Centre for Environment and Human Health, University of Exeter Medical School, Truro, UK; ${ }^{2}$ George Alleyne Chronic Disease Research Centre, University of the West Indies, Bridgetown, Barbados; ${ }^{3}$ MRC Epidemiology Unit, University of Cambridge, Cambridge, UK; ${ }^{4}$ School of Public Health, Massey University, Wellington, New Zealand

\subsection{6/jech-2018-SSMabstracts. 130}

Background Small island developing states (SIDS) have some of the highest rates globally of obesity, diabetes and related non-communicable diseases. A diet of energy dense and highly processed foods over fruit, vegetables and fibre is a major determinant of this burden. The majority of SIDS lack food sovereignty, are vulnerable to food insecurity and rely heavily on food imports. The need to increase local food production, particularly of non- or minimally processed foods, is seen as a pre-requisite to effective long term NCD prevention. This research aimed to explore what is known on community-based food production initiatives (CFPIs) in SIDS, including their number and distribution, characteristics, and their positive and negative impacts on health, social and economic wellbeing and the environment.

Methods A systematic scoping review was undertaken to identify literature from a range of disciplines. We searched 12 electronic databases for articles published in the last 20 years which concerned local food production initiatives in SIDS. In total, 8215 articles were screened and data abstracted for 153 eligible articles by two independent reviewers and verified by a third. Articles were included if they were set in one of 57 SIDS countries and reported health, social, economic or environmental impacts of or on CFPIs. Data abstraction and analysis focused on geographical location, type of CFPI, 